\title{
Effectiveness of Dual-Maneuver Using K-Wire and Dingman Elevator for the Reduction of Unstable Zygomatic Arch Fracture
}

Hyungwoo Yoon ${ }^{1}$, Jiye Kim ${ }^{1}$, Seum Chung ${ }^{2}$, Yoon-Kyu Chung ${ }^{1}$

${ }^{1}$ Department of Plastic and Reconstructive Surgery, Yonsei University Wonju College of Medicine, Wonju; ${ }^{2}$ National Health Insurance Corporation Ilsan Hospital, Ilsan, Korea

No potential conflict of interest relevant to this article was reported.

\begin{abstract}
Background: The zygoma is the most prominent portion of the face. Almost all simple zygomatic arch fractures are treated in a closed fashion with a Dingman elevator. However, the open approach should be considered for unstable zygomatic arch fractures. The coronal approach for a zygomatic arch fracture has complications. In this study, we introduce our method to reduce a special type of unstable zygomatic fracture. Methods: We retrospectively reviewed zygomatic arch view and facial bone computed tomography scans of 424 patients who visited the Wonju Severance Christian Hospital from 2007 to 2010 with zygomaticomaxillary fractures, among whom 15 patients met the inclusion criteria.

Results: We used a Dingman elevator and K-wire simultaneously to manage this type of zygomatic arch fracture. Simple medial rotation force usually collapses the posterior fractured segment, and the fracture becomes unstable. Thus, the posterior fracture segment must be concurrently elevated with a Dingman elevator through Keen's approach with rotation force applied through the K-wire. All fractures were reduced without any instability using this method.

Conclusion: We were able to reduce unstable and difficult zygomatic arch fractures without an open incision or any external fixation device.
\end{abstract}

Keywords: Zygomatic fractures / Facial injuries / Maxillofacial injuries

\section{INTRODUCTION}

The zygoma is the most prominent portion of the face and is commonly injured during facial trauma [1]. The zygomatic arch is a component of the zygomatic complex and makes up the part of anterior-lateral region of the face [2]. Isolated arch fractures comprise $10 \%$ of all zygoma fractures and 5\% of all facial bone fractures [3]. Treatment of zygomatic arch fractures varies from observation to open reduction. Some methods provide better aesthetic results through transcutaneous access using a hook, cu-

Correspondence: Jiye Kim

Department of Plastic and Reconstructive Surgery, Wonju Christian Hospital, Yonsei University Wonju College of Medicine, 20 Ilsan-ro, Wonju 220-701, Korea

E-mail: gen80@yonsei.ac.kr

Received June 30, 2014 / Revised July 23, 2014 / Accepted August 1, 2014 taneous access through the temporal region (Gillies' technique), or intra-oral access (Keen's technique) [4]. Almost all simple zygomatic arch fractures are treated in a closed fashion with a Dingman elevator using the Gillies' or Keen's approach [5]. However, complicated and unstable arch fractures require open reduction, and many studies have reported open methods to be more effective [6]. However, there are many difficulties and risks associated with an open approach. The coronal approach carries the risk of leaving a long scar, scalp numbness, alopecia, or temporal hollowing in addition to the usual risks associated with long operation time [7]. In this article, we introduce a method that does not use an open approach but is useful in reducing a special type of unstable zygomatic arch fracture. 


\section{METHODS}

We retrospectively reviewed 424 patient zygomatic arch views and facial bone computed tomography scans who visited our hospital from 2007 to 2010 with zygomaticomaxillary fractures. Only 15 cases fell into our specific category-cases in which the fracture segment was laterally rotated and the lateral margin of the fractured segment was more posterior than the typical zygomaticomaxillary fracture and the posterior segment was impacted under the posterior zygoma border (Fig. 1). This type of fracture develops from bidirectional force; and as such, an additional reduction force is needed apart from the Kirschner-wire on the malar prominence.

Patients were admitted to surgery under general anesthesia for

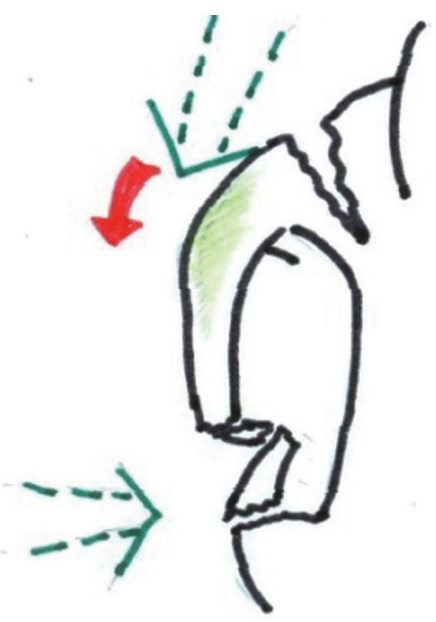

Fig. 1. Configuration of unstable zygomatic arch fracture. Dotted arrow, trauma force; red arrow, rotation vector.

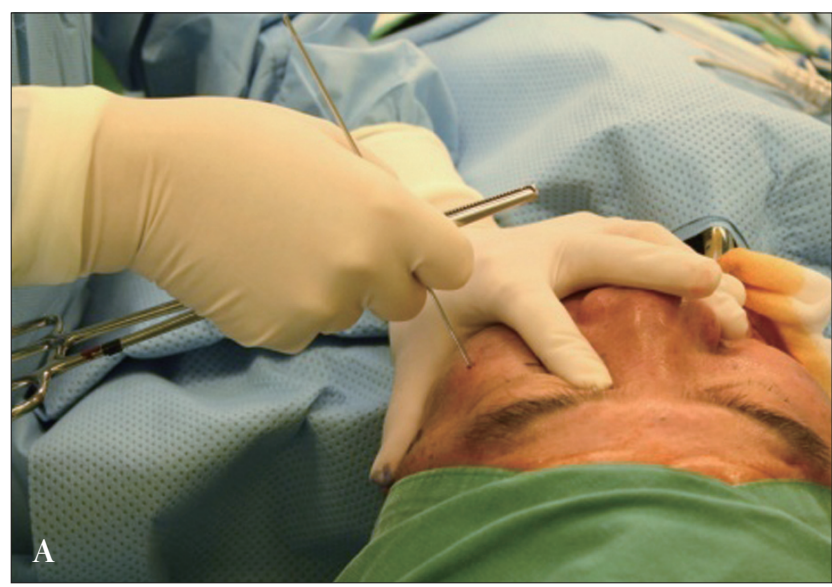

reduction and fixation of the fracture. We used a Dingman elevator and a K-wire to manage our specific type of zygomatic arch fracture (Figs. 2, 3). Dingman elevator was used through intraoral approach. Plate fixation was not used. The K-wire was inserted percutaneously at the malar prominence between the arch and body of zygoma. The malar prominence was determined by the intersectional point of the Frankfort horizontal line and the oblique vertical line between the lateral palpebral fissure and corner of the mouth [8]. All of the K-wires used were between $1.2 \mathrm{~mm}$ and $1.5 \mathrm{~mm}$ in diameter and were removed prior to the end of operation. After the operation, reduced arch and zygoma were protected by applying customized cutting paper cup.

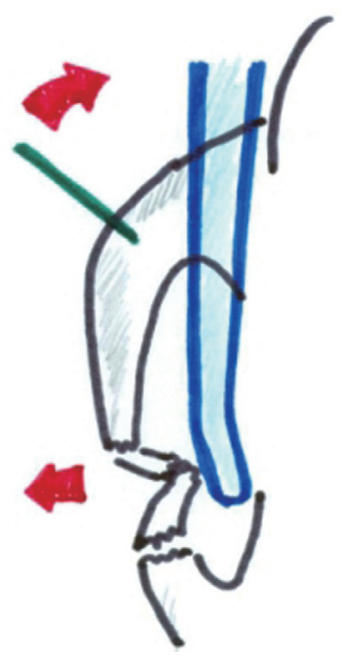

Fig. 2. Dual maneuver using K-wire and Dingman elevator. Red arrow, rotation vector; green line, inserted K-wire on zygoma segment; blue device, Dingman elevator.

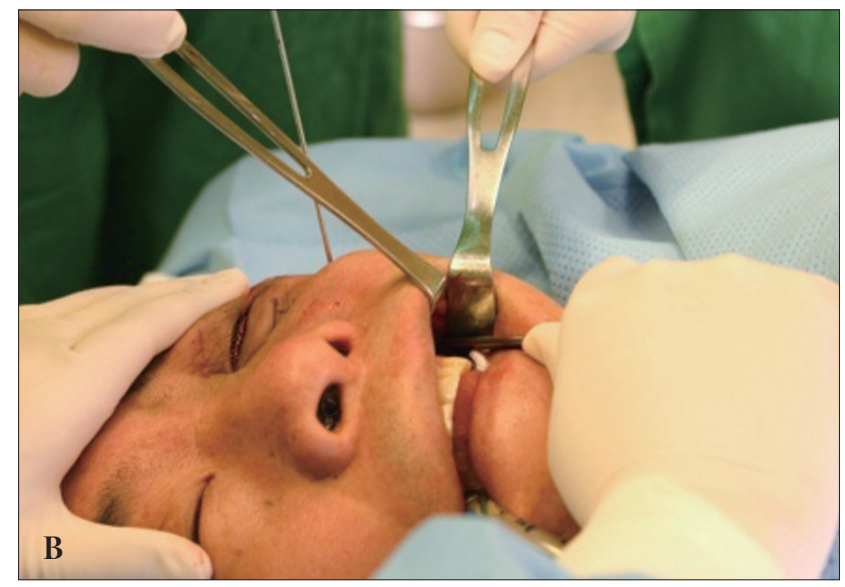

Fig. 3. Reduction method. (A) Reduction using K-wire. (B) Reduction using Dingman elevator and K-wire. 


\section{RESULTS}

We used a Dingman elevator and K-wire simultaneously to manage this type of zygomatic arch fracture. Simple medial rotation force usually collapses the posterior fractured segment, and the fracture becomes unstable. Thus, the posterior fracture segment must be concurrently elevated with a Dingman elevator through Keen's approach with rotation force applied through the K-wire. All fractures were reduced without any instability using this method (Fig. 4). We did not need an open method. No complications occurred in the immediate postoperative period, and there was no injury to the facial nerve.

\section{DISCUSSION}

Treatment of zygoma fractures varies from observation to Kirschner-wire fixation and open approaches such as mini-plate fixation. Almost all simple zygomatic arch fractures are treated in a closed fashion with a Dingman elevator using Gillies' or Keen's approaches [5]. More extensive access is required when there is a need for osteosynthesis, which may involve an open preauricular or coronal approach or even an extension of infraorbital access. An unstable and complicated arch fracture also requires an open method [6]. Although open methods are more effective, the coro- nal approach carries the risk of leaving a long scar, scalp numbness, alopecia, or temporal hollowing in addition to the usual risks associated with a long operation time. In the study, the operation time of dual method was similar to or slightly longer than the Gillies' procedure and was much shorter than the time of open surgery. Thus, closed reduction techniques represent the traditional method for nearly all zygomatic fractures.

However, closed reduction of all arch fractures may result in incomplete reduction or more unstable fractures, leading to unsatisfactory cosmetic outcomes and persistent functional disturbances. A reasonable application of different treatment methods, according to careful assessment of the fracture types, is necessary for successful outcomes.

A zygomatic arch fracture is the result of an assortment of traumas, and the nature of each fracture varies according to the type of injury involved, including whether it was a low energy or high energy injury, the result of falling down, or due to a traffic accident [4]. These traumas may cause isolated zygomatic arch fractures or zygomaticomaxillary complex fractures due to one-vector force or unstable zygomaticomaxillary complex factures due to twovector force. In severe cases, the trauma may result in a comminuted arch fracture. In any case, variations in force vectors result in a spectrum of fracture types. Most commonly, one-vector injuries result in simple type fractures such as M-shape arch fractures
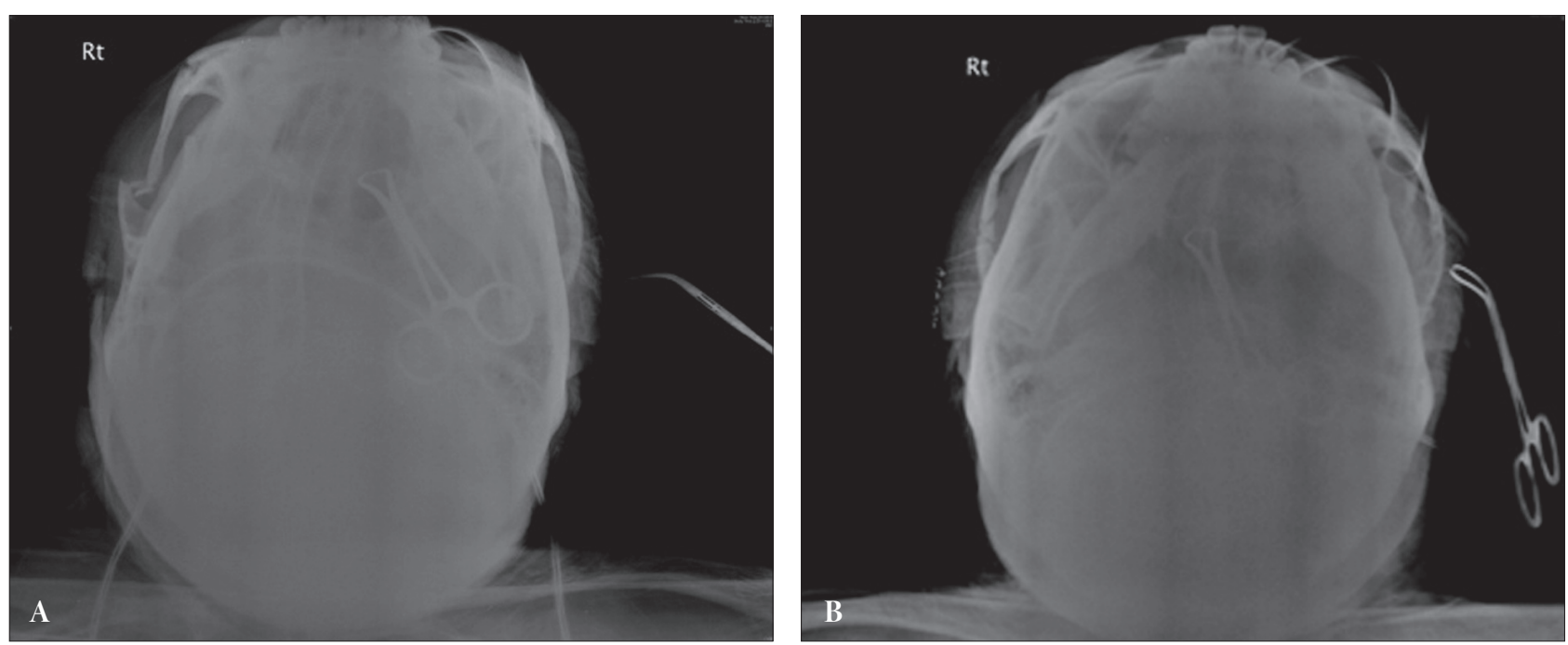

Fig. 4. A 40-year-old man with right zygomatic arch fracture. (A) Preoperative photograph. (B) Immediate postoperative photograph. 
and can normally be treated by closed reduction.

In this study, the type of fractures examined was the result of trauma from at least two vectors, particularly those leading to lateral rotation of the malar prominence where the lateral margin of the fractured segment was more posterior than in typical cases of zygomaticomaxillary fractures and also where the posterior segment was impacted under the posterior zygoma border. In these cases, prior treatment methods involving access through the temporal region (Gillies' technique) or intra-oral access (Keen's technique) may be inadequate to achieve complete reduction [4]. While these unstable fractures can be reduced through open surgery, we determined that shortcomings of open reduction could be overcome by applying a dual-maneuver reduction with a Kirschner-wire and a Dingman elevator. The Kirschner-wire is placed percutaneously at the junction between the arch and the body of the zygoma, allowing for medial rotation of the fractured segment to the malar prominence. Therefore, Dingman elevator insertion using Keen's technique was possible and more efficient reduction was accomplished.

The use of Kirschner-wire for surgical reduction of zygomatic arch fractures has already been introduced in 2010 [9]. Recent reports have favored percutaneous Kirschner-wire fixation as a less invasive technique for simple and complicated zygoma fractures. The combination of Gillies' elevation with one-point percutaneous Kirschner wire fixation of isolated simple zygoma fractures was effective in restoring preinjury appearance and function and avoiding soft tissue morbidity.
We conclude that the dual-maneuver with Kirschner-wire and Dingman elevator for the specific type of unstable fracture results in successful reduction without requiring open surgery.

\section{REFERENCES}

1. Mezitis M, Stathopoulos P, Rallis G. Use of a curved mosquito for reducing isolated zygomatic arch fractures. J Craniofac Surg 2010;21: 1281-3.

2. Gulicher D, Krimmel M, Reinert S. The role of intraoperative ultrasonography in zygomatic complex fracture repair. Int J Oral Maxillofac Surg 2006;35:224-30.

3. Swanson E, Vercler C, Yaremchuk MJ, Gordon CR. Modified Gillies approach for zygomatic arch fracture reduction in the setting of bicoronal exposure. J Craniofac Surg 2012;23:859-62.

4. de Santana Santos T, da Rocha Neto AM, Medeiros R Jr, Antunes AA, de Oliveira DM. Treatment of zygomatic arch fracture with lag screws. J Craniofac Surg 2011;22:1468-70.

5. Comert E, Comert A, Uz A, Tuncel U, Elhan A. Anatomic basis of percutaneous Kirschner wire insertion in zygoma fractures. J Craniofac Surg 2011;22:1483-5.

6. Czerwinski M, Martin M, Lee C. Quantitative comparison of open reduction and internal fixation versus the Gillies method in the treatment of orbitozygomatic complex fractures. Plast Reconstr Surg 2005;115:1848-54.

7. Islamoglu K, Coskunfirat OK, Tetik G, Ozgentas HE. Complications and removal rates of miniplates and screws used for maxillofacial fractures. Ann Plast Surg 2002;48:265-8.

8. Lew DH, Park BY, Lee HB, Lew JD. Simple fixation method for unstable zygomatic arch fracture using double Kirschner's wires. Plast Reconstr Surg 1998;101:1351-4.

9. Park BY, Song SY, Yun IS, Lee DW, Rah DK, Lee WJ. First percutaneous reduction and next external suspension with Steinmann pin and Kirschner wire of isolated zygomatic fractures. J Craniofac Surg 2010;21:1060-5. 